This is the Accepted Version of the article: Haolin Zhang, Angel Lizana, Claudio lemmi, Freddy A. Monroy-Ramírez, Andrés Marquez, Ignacio Moreno, Juan Campos, "Self-addressed diffractive lens schemes for the characterization of LCoS displays ," Proc. SPIE 10555, Emerging Liquid Crystal Technologies XIII, 105550I (8 February 2018).

Available at DOI: $\underline{\text { https://doi.org/10.1117/12.2290434 }}$

(C) 2018 Society of Photo Optical Instrumentation Engineers (SPIE). One print or electronic copy may be made for personal use only. Systematic reproduction and distribution, duplication of any material in this publication for a fee or for commercial purposes, or modification of the contents of the publication are prohibited. 


\title{
Self-addressed diffractive lens schemes for the characterization of LCoS displays
}

\author{
Haolin Zhang*a, Angel Lizana ${ }^{\mathrm{a}}$, Claudio Iemmi ${ }^{\mathrm{b}}$, Freddy A. Monroy-Ramírez ${ }^{\mathrm{c}}$, Andrés Marquez ${ }^{\mathrm{d}}$, \\ Ignacio Moreno ${ }^{\mathrm{e}}$, Juan Campos ${ }^{\mathrm{a}}$ \\ a Departamento de Física, Universitat Autònoma de Barcelona, Bellaterra, 08193, Spain; ${ }^{\mathrm{b}}$ \\ Universidad de Buenos Aires, Facultad de Ciencias Exactas y Naturales, Departamento de Física, \\ Consejo Nacional de Investigaciones Científicas y Técnicas, Buenos Aires, Argentina; ${ }^{\mathrm{c}}$ \\ Departamento de Física, Universidad Nacional de Colombia - Sede Bogotá Carrera 45 No 26-85,

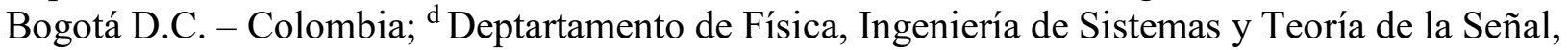 \\ Universidad de Alicante, Ap. 99, 03080 Alicante, Spain; ${ }^{e}$ Departamento de Ciencia de Materiales, \\ Óptica y Tecnología Electrónica, Universidad Miguel Hernández de Elche, 03202 Elche, Spain
}

\begin{abstract}
We proposed a self-calibration method to calibrate both the phase-voltage look-up table and the screen phase distribution of Liquid Crystal on Silicon (LCoS) displays by implementing different lens configurations on the studied device within a same optical scheme. On the one hand, the phase-voltage relation is determined from interferometric measurements, which are obtained by addressing split-lens phase distributions on the LCoS display. On the other hand, the surface profile is retrieved by self-addressing a diffractive micro-lens array to the LCoS display, in a way that we configure a Shack-Hartmann wavefront sensor that self-determines the screen spatial variations. Moreover, both the phase-voltage response and the surface phase inhomogeneity of the LCoS are measured within the same experimental set-up, without the necessity of further adjustments. Experimental results prove the usefulness of the above-mentioned technique for LCoS displays characterization.
\end{abstract}

Keywords: Liquid Crystals, Calibration, Holographic display, Surface Measurement

\section{INTRODUCTION}

Liquid Crystal Displays (LCDs) is a mature technology widely applied in different applications. For instance, to eliminate the effect of air turbulences introduced by a large telescope system [1], as adaptive optics elements to correct the wavefront aberration [2, 3], in interferometer based metrology systems to control the phase distribution [4], or in wavelength selective systems to manipulate the lightwaves [5-7]. LCDs are also extensively used as spatial light modulators (SLMs) to generate different diffractive optical elements (DOEs) for dynamic processes [8, 9]. In this framework, they provide the feasibility to obtain structured illumination $[10,11]$, or they stand as pattern generators to achieve real-time laser beam shaping $[12,13]$. Last but not least, LCDs are also used to implement optical tweezers $[14$, 15], optical encryption [16] or digital lenses with improved performance $[17,18]$.

Liquid Crystal on Silicon (LCoS) displays [19] are reflective LCDs which provide numbers of advantages compared with transmissive LCDs, such as high resolution, small pixel size, and very appealing fill factor. What is more, as light performs a double pass within the LCoS displays due to the reflection configuration, they present a larger phase modulation than transmissive devices with a same thickness. For an accurate control of those devices, an effective calibration and optimization of their phase-voltage response as well as of the screen phase inhomogeneity is required for their efficient performance.

Under this scenario, numbers of LCoS calibration methods [20-28], mainly based on interferometry [22-24] or diffraction $[25,26]$, are proposed in literature. In the case of reflective LCoS displays, diffractive based methods are not suitable to be applied within calibration processes due to the time-fluctuations of the phase phenomenon [21]. Hence, alternative techniques were proposed to minimize the phase fluctuations effect and prevent the DOEs efficiency loss related to such phenomenon [27-33].

Another critical drawback of the LCoS displays is the spatial phase inhomogeneity, which is related to spatial variations of the display flatness. The spatial inhomogeneity, mainly introduced by screen lateral stresses or glass 
thickness variations during the fabrication, presents an extra spatial phase distribution that degrades the performance of the modulator. Thus, to a proper phase modulation performance of LCoS devices, this extra phase distribution must be calibrated and corrected. As a consequence, liquid crystal screen uniformity is measured from different experimental strategies in order to compensate the phase distribution incorrectness [34-36].

Recently, LCD displays self-calibration methods, at which the information of the LCD is retrieved by selfaddressing different DOEs on the same SLM, were proposed [29]. The self-calibrating approach provides an obvious advantage compared to standard calibrating methods by avoiding the usage of additional optical elements in the optical scheme (i.e., without requiring commonly used external interferometry or diffraction based optical arrangements). In fact, the same LCD to be calibrated is employed to display the digital optical elements that allow the device calibration. In particular, J. L. Martinez et al. [29] evaluated the overall averaged phase modulation of an LCoS display by selfaddressing two diffractive elements simultaneously displayed on it. To do so, the screen of the LCoS was divided in two halves in which a uniform image ('piston') and a symmetric binary phase grating were addressed, respectively. By adding different constant gray levels to the 'piston' image and selecting a proper diffractive order generated by the grating image, an interferometric pattern was achieved. In fact, the first half acted as a phase-shifting mirror and the second half acted as a tilted reference plane-wave that interfered with the wave coming from the 'piston'. Note that different gray levels added to the piston (i.e., different "mirror" shifts) led to different displacements of the interference pattern, from which the overall phase modulation of the device was determined. Unfortunately, their approach does not provide the surface phase inhomogeneity (deformation of the screen flatness) which is a critical LCoS calibration factor.

We present in this paper an alternative self-calibration method based on addressing diffractive lens configurations, valid to both characterize the overall phase-gray level performance and the LCoS screen profile. In particular, a split-lens configuration [11] and an arrangement of dynamic lenses (Shack-Hartmann wavefront sensor configuration) are proposed, respectively. The overall phase distribution as a function of the addressed voltage is retrieved by the implementation of a split-lens configuration, which creates a fringes-like interferences system from which the phasevoltage curve can be determined. Furthermore, the screen profile of the display is determined by self-addressing a diffractive lens array that implements a Shack-Hartmann wavefront sensor that self-measures the screen profile. We want to emphasize that the two above-mentioned lens schemes are employed in the same spatial light modulator and both selfmeasurements can be performed without the modification of the experimental set-up. The proposed calibration method provides three main advantages compared to other existing techniques [11, 15, 20,29]. First, the split-lens configuration provides the phase-voltage look-up table even in presence of time-fluctuations of the phase phenomenon, as we are dealing with an interferometric system, and the required average phase is determined as a function of the applied voltage. Second, both the phase-voltage performance and the phase profile of the display can be acquired within the same optical arrangement by generating two diffractive lens configurations (split lens schemes [11] or Shack-Hartmann configurations $[34,38,39]$ ) on the same LCoS display. Third, the LCoS features can be self-retrieved without the implementation of any external calibrating set-ups (as it is the common case), which leads to a more compact system.

The outline of this work is as follows. In section 2, the method for the self-calibration of the LCoS phase-voltage curve is described. In section 3, the technique to perform the self-measurement of the LCoS screen phase profile is proposed. In this section, we thoroughly discusss the generation of the microlens array and the ulterior retrieving of the screen profile from the obtained light spot array. Next, in section 4, we experimentally implemented the self-calibration methods discussed in sections 2 and 3, and both the phase-voltage curve and the surface profile of a particular LCoS display are measured. At last, the main conclusions are given in section 5.

\section{SELF-CALIBRATION OF THE LCOS PHASE-VOLTAGE PERFORMANCE BASED ON A SPLIT-LENS CONFIGURATION}

To efficiently generate DOEs with LCoS displays, the phase modulation performance of such devices must be calibrated. In this section, we describe a method for the calibration of the mean phase of the LCoS as a function of voltage (gray level), by using a split-lens configuration.

The self-calibration methodology consists in addressing a certain phase distribution to the LCoS, allowing us to create a controlled interference pattern to a propagated plane. In our method, the interference is achieved by sending aphase distribution generating a two-sectorial split-lens configuration. In particular, the generated split-lens configuration implements a diffractive lens split in two halves to a certain distance $a$. Each of the two split-lens sectors focuses the incident light to the focal plane at two distinctive focal points. Hence, in the far field, the two propagated light beams coming from the focal points at the focal plane produce an interference pattern. This situation can be approximated to the coherent superposition of two plane-waves with different propagation directions, which provides a fringes-like pattern. 
We want to emphasize that even though the addressed split-lens scheme implements a DOE equivalent to a lens split in two halves, the phase distribution sent to the LCoS is continuous. In such a way, the non-desired contribution of the light passing through the hole left by the split-lens, occurring in real lens based systems, is avoided in the LCoS based implementation. Another advantage of the LCoS generated split-lens stands as the parameters of the split-lens configurations can be changed digitally, such as the focal length of the lenses or the distance between the two halves, which allow us to control the period of the created fringes pattern.

The scheme of the split-lens optical implementation is presented in Fig. 1. We use a linearly polarized, coherent and collimated light beam to illuminate the optical system. Afterwards, the input light meets the SLM, where we have addressed the phase distribution corresponding to the two split-len sectors with a separation distance of $a$ (see inset image (a) in Fig. 1). Hence, two focal points separated to a distance of $A=a$ (represented as $F 1$ and $F 2$ in Fig. 1) are obtained at the $S$ plane (focal plane of the split-lens). The two coherent light sources at $F 1$ and $F 2$ have the same intensity and their propagation produce an interference pattern to a propagated field (beyond the $B$ plane in Fig. 1). At last, by introducing a convergent lens with a certain magnification, the interference pattern at the $I$ Plane (see inset image (b)) is imaged and recorded by the CCD (inset image(c)). At last, the angular separation between the two spots (angle $\alpha$ in Fig. 1) depends both on the $a$ and $f_{1}$ parameters; thus, a digital controllable interference pattern is easily realized just by tuning these two parameters $\left(a\right.$ and $\left.f_{1}\right)$.

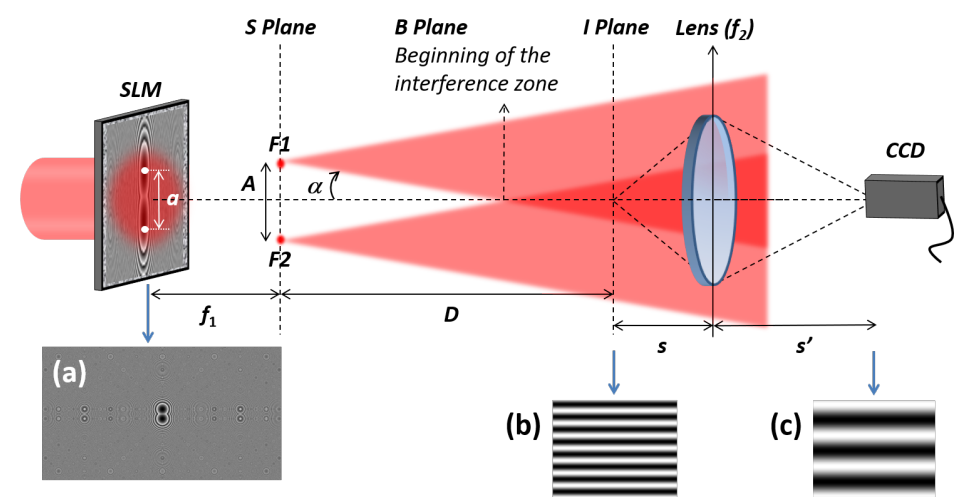

Fig. 1. Scheme of the optical set-up used to perform the phase-voltage calibration of the SLM.

Even though Fig. 1 is provided in a transmissive scheme for the sake of clarity, the real experimental implementation is conducted into a reflection sketch because the LCoS used is a reflective element. Hence, in the real optical system, a beam-splitter (B-S) is introduced to receive the reflected image and a flat reflective mirror is used to compress the optical track in order to achieve a more compacted system. The implemented experimental set-up is presented in section 4 .

Once we obtain the interference pattern from the above-stated system, the phase-voltage LCoS performance can be retrieved. We firstly generate a phase distribution representing the split-lens pattern (e.g., image (a) in Fig. 1). Afterwards, the phase values for one of the two LCoS halves (i.e., for one of the two bi-lens sectors) is modified by adding a particular constant gray level, while the other half sector is not modified. In this case, the interference pattern recorded in the CCD is vertically shifted to a certain distance, which is directly related to the gray level added. The phase distribution to be addressed to the two LCoS halves can be written, respectively as [16]:

$$
\begin{gathered}
U_{1}=\exp \left[i \frac{\pi}{\lambda f}\left(r^{2}+a_{0}{ }^{2}-2 r a_{0} \cos \left(\theta_{i}-\theta_{0}\right)\right)\right], \\
U_{2}=\exp \left[i \frac{\pi}{\lambda f}\left(r^{2}+a_{1}{ }^{2}-2 r a_{1} \cos \left(\theta_{i i}-\theta_{1}\right)+\phi(V)\right)\right],
\end{gathered}
$$

where $\lambda$ and $f$ are the wavelength of the input light and the focal length of the two split-lens sectors.. Moreover, $r$ represents the radial coordinate in the plane of the lens; $a_{0}$ and $a_{1}$ are the distances of the two lens sector centers to the origin of the coordinate system; $\theta_{0}$ and $\theta_{1}$ are the angular positions of the two sector centers $\left(\theta_{0}=\pi / 2\right.$ and $\theta_{1}=3 \pi / 2$ for the particular case shown in Fig. 1) and $\theta_{i}$ and $\theta_{i i}$ are restricted into the ranges $[0, \pi)$ and $[\pi, 2 \pi)$. Note that in Eq. (2), a uniform phase value $\phi(V)$, related to a given gray level, is added to the phase term. Therefore, by changing the input 
voltage of the LCoS, the phase term in Eq. (2) is correspondingly modified. According to the relations in Eqs. (1) and (2), the complete phase distribution function to be addressed at the SLM can be written as,

$$
U_{N=2}(r, \theta)=U_{1}+U_{2} \text {. }
$$

After addressing this phase-distribution (Eq. (3)) to the LCoS display, the split-lens scheme is implemented and two focal spots are created at the focal plane. After propagation, the two corresponding waves interfere. As a consequence of the addition of a constant gray level in the $U_{2}$ term in Eq. (3), a transversal shift in the corresponding fringes pattern is produced. This is due to the phase-difference variation associated to the addition of the extra gray level. Hence, by providing a whole gray level range (0-255) in the second section, the relation between the average phases versus addressed gray level can be retrieved from the corresponding fringes displacements, by using simple calculations, as those provided in Ref. [21].

\section{SELF-CALIBRATION OF THE LCOS SCREEN SURFACE BY USING A MICRO- LENS ARRAY}

In order to achieve an optimal performance of the LCoS display, we not only need to calibrate the phase-voltage curve, but also to determine the LCoS display surface inhomogeneities, as they usually present an extra phase distribution at the screen. This spatial phase variation, that we call zero-phase term (ZPT), is usually introduced by mechanical stresses on the screen, and it should be corrected. We proposed in this section a method for the selfcalibration of the ZPT of SLMs, which is based on the implementation of a self-addressed micro-lens array (SharkHartmann wavefront sensor). From the calibration of the ZPT, the compensation of the SLM surface inhomogeneities can be conducted.

Shack-Hartmann (S-H) wavefront sensors refer to optical instruments consisting of a micro-lens array with the same focal length. They provide a feasible ability to measure the phase distribution of input beams or the spatial aberrations generated by unideal optical devices. In this case, each small microlens focuses the fraction of the income light into its own focal point. If the income wavefront is ideally flat, the light spots at the focal plane should have a same separation distance from the neighbouring focal spot (i.e., they focalize at their corresponding centers). However, if the input wavafront carries some aberrations, the light spots on the focal plane present certain offset displacements from their ideal spots. Thus, by measuring the displacements of each focal point from their ideal positions, the input wavefront can be retrieved by implementing proper numerical algorithms [37-39].

In our case, the S-H micro lens array is digitally implemented in the LCoS display to be calibrated. If the input wavefront is perfectly flat, the corresponding dots distribution at the focal plane will carry information of the ZPT of the studied LCoS. . In order to obtain the spot array at the focal plane, a collimated and linearly polarized light beam is used to illuminate the LCoS display panel, where the S-H scheme is addressed. In particular, the optical arrangement used to implement the LCoS screen calibration method is equivalent to that in Fig. 1, but now the S-H configuration is addressed, as shown in Fig. 2.

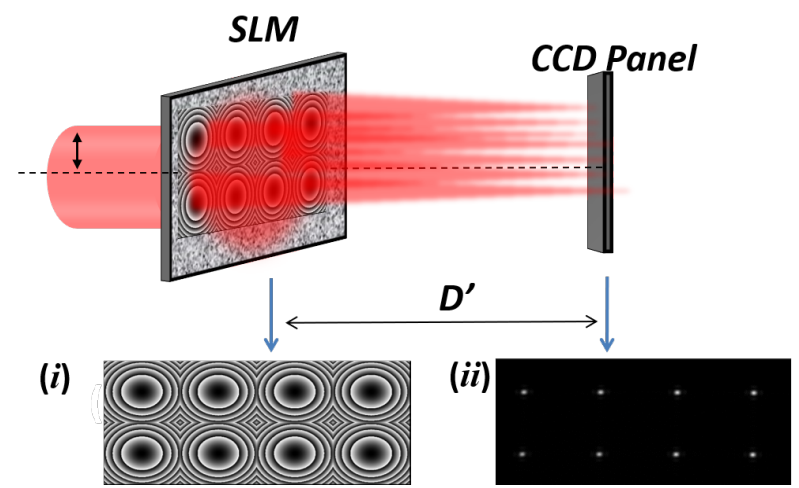

Fig. 2: Optical scheme used to self-calibrate the screen profile of the LCoS. Inset images: (i) Phase distribution sent to the LCoS to generate a microlens array (Shack-Hartmann configuration); and (ii) Corresponding intensity distribution at the focal plane. 
Figure 2 schematizes a $4 \times 2$ microlenses configuration (see inset image (i) in Fig. 2) addressed to the LCoS display. A CCD camera is placed at the focal plane of the micro-lens array (to a distance $D^{\prime}$ from the SLM) to record the corresponding focal points distribution (represented in the inset image (ii)). We want to note that we chose a dimension of $400 \times 400$ pixels for each micro-lens because as larger the microlens aperture is, the higher the efficiency for the generated diffractive micro-lens. In the chosen configuration, the whole $4 \times 2$ microlenses pattern possess a dimension of $1600 \times 800$ pixels. We implemented the whole micro-lens array in a PLUTO LCoS display with a dimension of $1920 \times 1080$ pixels. Hence, the generated microlenses array pattern does not cover the full LCoS screen panel. This limitation is solved by performing a screen scanning process by conducting 8 displacements of the microlenses array with steps of 50 pixels in both the $x$ and $y$ directions. The intensity of the spots distribution is recorded in the CCD camera for each conducted displacement, and by combining all the recorded images, a final $32 \times 16$ points image is obtained. For the sake of clarity, a video presenting the scanning process is provided as Video 1 , and the final intensity pattern obtained is shown in Fig. 3.
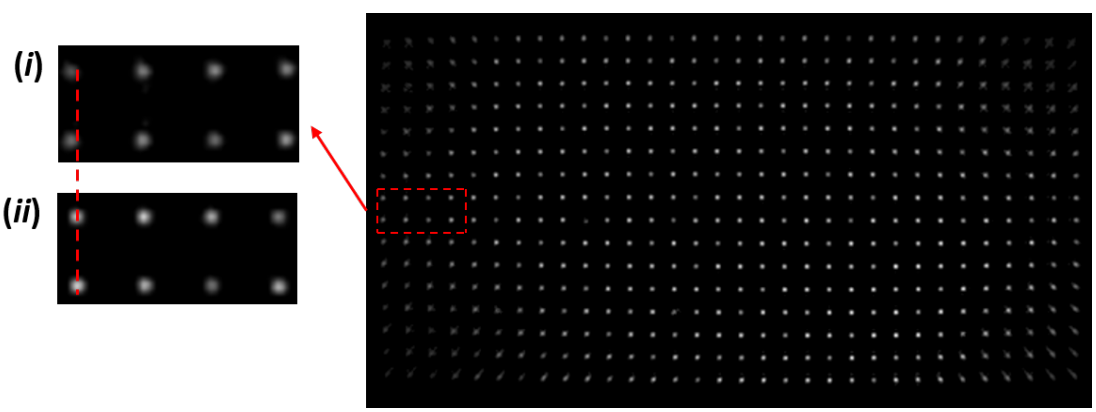

Fig. 3: Light spots pattern after scanning the full LCoS screen by using 8 displacements of the 4x 2 lens array in both $x$ and $y$ directions (see Video 1). Inserted images: (i) Spot distribution of a certain selected section at the edge; (ii) Ideal distributed light spots corresponding to the spots of image $(i)$.

Image in Fig. 3 shows the dot distribution obtained when applying the above-stated method on a particular LCoS display. Even though light spots in the central region of Fig. 3 are almost equidistant, the spots at the edges show an evident deviation from their theoretical centers, which indicate a phase-distortion in the LCoS screen. Note that as we are illuminating the system with an almost plane wave, the observed distortion is mainly generated by the LCoS surface inhomogeneities. As an example of the captured spatial distortion, we amplify a certain dots-section in the left- middle corner (inset image $(i)$ in Fig. 3). For comparison, the spot distribution of the Shack-Hartmann wavefront sensor corresponding to a perfectly flat LCoS display (ideal case) is also shown in the inset image (ii). The offsets of the light spots between the distorted pattern, inset $(i)$, and the ideal pattern, inset (ii), are highlight by the red-dashed line. Note that each light spot in the CCD is generated by the corresponding phase distribution of one micro-lens in the LCoS (see Fig. 2(i)), thus, each light spot carries the surface information of the corresponding section in the LCoS. Next, by considering the offset distance of the ideal light spot and the real light spot, as well as the focal length of the microlenses spot, the tilt angle of the certain section is calculated. Under this scenario, a discrete function for the LCoS surface derivatives can be obtained. From this data, by using an integration method, the ZPT of the screen profile can be retrieved. Finally, a continuous ZPT function can be determined by using interpolation methods.

At this point, it is worthy to discuss how to determine the ideal light spot array positions, from which the corresponding deviations are measured. As it is mentioned above, if a light spot array is obtained from a perfectly flat LCoS display, each light spot will be focussed at its coordinate center, and they will share the same distance in $x$ and $y$ directions with their neighbouring spots. Under this configuration, we can define a square grid to which each square sets its corresponding light-dot at its center, this being the reference-grid. Thus, a squared reference-gridis always added to the measured light spot patterns, to determine the dots displacements. In particular, the deviation of each light spot from its corresponding square-center, into the reference-grid, is calculated to determine the LCoS screen derivatives function.

In order to accurately determine the light spots deviations by using the squared grid approximation, a reasonable selection of the grid spatial position, as well as of the size and the number of the grid squares must be done. In our case, as the grid-coordinate origin is not known, we arbitrarily placed the grid over the final $32 \times 16$ light spots image. This arbitrary selection of the grid spatial position leads to different error sources that must be discussed. First, a possible $x-y$ displacement of the reference grid from the ideal position can be produced. However, this situation is not important in terms of surface profile determination. In fact, a given $x-y$ displacement adds a constant value to each deviation measure. 
This constant introduced becomes a linear phase after numerical integration, which introduces a tilt in the retrieved ZPT but does not modify its phase profile.

On the other hand, differences between the selected grid squares size with the theoretical one may dramatically influence the LCoS profile calculation. In fact, the grid square size error introduces a linear displacement error in the deviation measurements. This situation adds an artificial quadratic error to the retrieved screen profile after numerical integration. Hence, an accurate grid square size estimation must be conducted, for instance, by considering the particular relations between the CCD camera and the LCoS display. As above-mentioned, the scanning step set along the LCoS display is of 50 pixels and the pixel size of the particular LCoS used is of $8 \mu \mathrm{m}$. So, the distance between the center of two neighbouring microlenses generated in the LCoS display is of $400 \mu \mathrm{m}(50 \times 8 \mu \mathrm{m})$. Moreover, the CCD camera used presents a resolution of $2048 \times 2048$ pixels, with a pixel size of $7.4 \mu \mathrm{m}$, and thus, a distance of $400 \mu \mathrm{m}$ in the CCD camera is equivalent to 54 pixels of the $\operatorname{CCD}(400 \mu \mathrm{m} / 7.4 \mu \mathrm{m}=54.05)$, this being the optimal size for the squares in the grating. . To fulfil the camera dimensions, a 38x38 grid was selected. The final reference-grid used for deviations metrology is shown in Fig. 4.

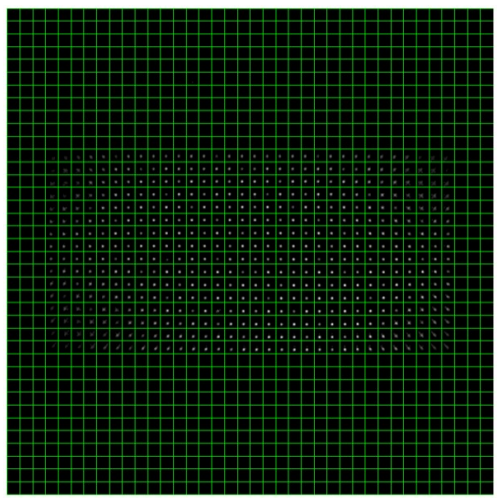

Fig. 4: $38 \times 38$ squared-grid in the CCD camera (highlighted in green) to determine the light spot deviations from their ideal positions.

As stated above, from the calculated dots-deviations, the LCoS display profile can be retrieved by using numerical integration. In order to achieve a continuous surface profile, a cubic spline interpolation was used to estimate the continuous surface of the calibrated LCoS.

\section{EXPERIMENTAL CALIBRATION OF THE LCOS DISPLAY}

We provide the experimental measurement of both the phase-voltage look-up table (section 2) and the LCoS surface profile (section 3) in this section. The experimental test is realized by using a single optical arrangement and by addressing two different phase distributions (split-lens and Shack-Hartmann wavafront sensor patterns) to the LCoS display. The experimental layout to provide the two calibrations is shown in Fig. 5(a). Moreover, in Fig. 5(b) we provide a detail of the calibrated LCoS display.

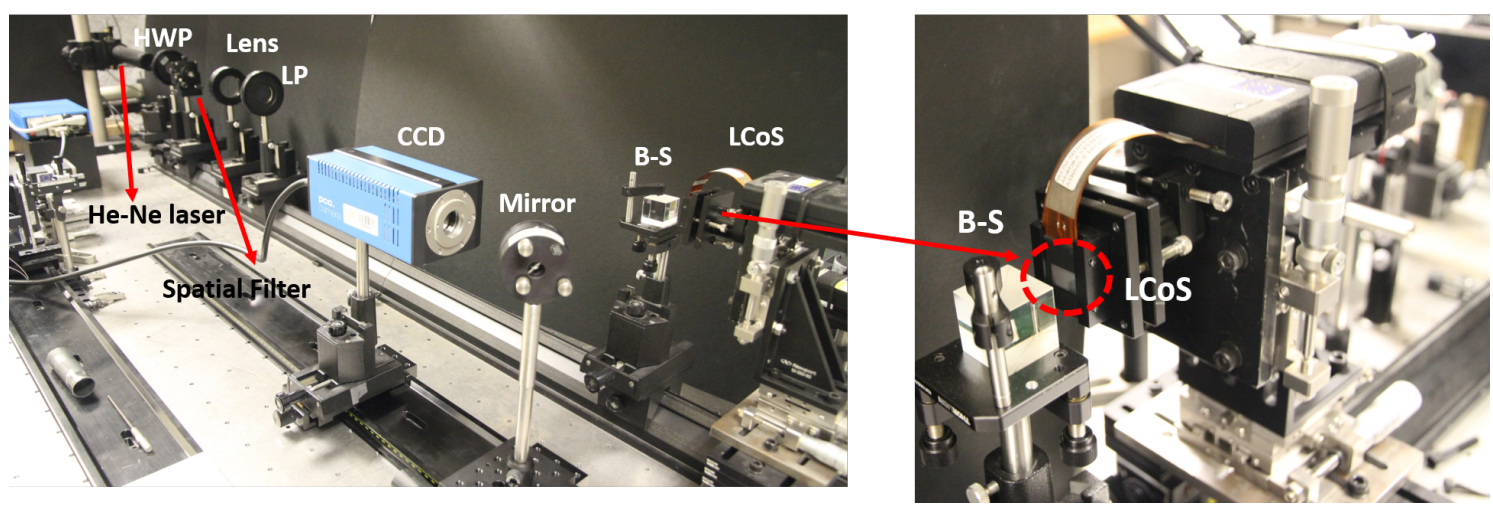


Fig. 5: Experimental set-up used for the LCoS characterization: (a) Experimental set-up to achieve the two calibrations; and

(b) Detail of the reflective screen of the LCoS under calibration.

In our experiment, the illumination is provided by a He-Ne laser working at $632.8 \mathrm{~nm}$. The light polarization is controlled by the combination of the half waveplate (HWP) and a linear polarizer (LP), allowing us to obtain a linear polarized light with controlled intensity. Later, light is filtered by means of a spatial filter, which consists of a microscope objective $(\times 20)$ and a pinhole. The output light from the pinhole is collimated by properly placing a convergent lens. Afterwards, in order to illuminate the system with wavefront as plane as possible, the quality of the collimated light is evaluated by a shearing interferometry device (SI050 shear-plate, distributed by Thorlabs). Afterwards, the controlled illumination reaches the LCoS display, distributed by HOLOEYE. Because the LCoS display is a reflective device, a beam-splitter (B-S) is introduced to set a reflective configuration. Finally, to compact the optical arrangement, a flat mirror steers the reflected light to the backward direction. Note that in our experiment, the LCoS display provided by HOLOEYE has a fill factor of $87 \%$, a reflectivity of $65-70 \%$, and diffraction efficiency of more than $80 \%$. As a CCD camera we use a PCO.2000 with a resolution of $2048 \times 2048$ pixels.

After the implementation of the experimental set-up, we measured the LCoS display phase-voltage look-up table and the surface profile by using the techniques provided in sections 2 and 3 . The experimental process and results are given in sub-sections 4.1 and 4.2 .

\section{A. LCoS display phase-voltage look-up table determination}

In this sub-section, we experimentally test the split-lens based technique described in section 2 . We sent to the LCoS display the phase distribution given in Eq. (3), and we captured the corresponding interference pattern with the CCD. The separation distance between the two halves was set to $0.2 \mathrm{~mm}$ and the focal length of the split-lens was set to 350 $\mathrm{mm}$. The phase pattern $\phi(V)$ in Eq. (2) was initially to set zero, and both $a_{0}$ and $a_{1}$ were set to $0.1 \mathrm{~mm}$. In specific, we implemented two split lens sectors symmetric displaced from the LCoS screen center. Afterward, we gradually modified the constant phase term $\phi(V)$ by adding a constant gray level, from 0 to 255 in steps of 8 gray levels, to all the pixels of the second split lens (Eq. (2)). Hence, 33 different interference intensity distributions were obtained in the CCD and they presented a horizontal displacement within each step of the added gray level. We set the interference pattern corresponding to the gray level 0 as the reference pattern. From this image, cross-correlation calculations were performed with the other interference patterns to determine the phase value associated to each gray level evaluated. Finally, we want to emphasize that the CCD integration time $(\sim 0.5$ second $)$ is set significantly longer than the typical LCoS phase-fluctuations period $(\sim 8 \mathrm{~ms})$. As a consequence, the used fringe patterns are averaged images, and the proposed calibration method provides the desired mean phase values.

Note that the split-lens separation distance can be dynamically modified, so we can control the period of the resulting fringes pattern at the CCD plane (see Fig. 1). To analyse the influence of this parameter in the accuracy of the phase-voltage determination, we also analysed two more cases with the split-lens separations as $0.4 \mathrm{~mm}$ and $0.5 \mathrm{~mm}$ within the same optical implementation. Moreover, each separation case was measured one hundred times to obtain the corresponding standard deviations. The phase-voltage curves for the three studied split-lens centers distances are represented in Fig. 6 as the green $(0.2 \mathrm{~mm}$ separation case), blue $(0.4 \mathrm{~mm}$ separation case) and red $(0.5 \mathrm{~mm}$ separation case) curves. Their corresponding standard deviations (represented as error bars in the figure) are also given. Note that all three curves in Fig. 6 show an approximate linear phase variation that ranges from 0 rad to $\sim 6.28$ rad. More importantly, the error bars associated to the calculated values provide further analysis about the measurements sensibility. In particular, the green curve in Fig. $6(0.2 \mathrm{~mm}$ case $)$, shows the largest standard deviations with a maximum error bar value of $0.98 \mathrm{rad}$, while the red curve $(0.5 \mathrm{~mm}$ case) shows smaller error bars with the maximum value of $0.28 \mathrm{rad}$. By contrast, the blue curve $(0.4 \mathrm{~mm}$ case $)$ shows the smallest standard deviation values, with a maximum error bar of 0.22 $\mathrm{rad}$. We hypothesize that the difference in the associated standard deviations obtained for the three-mentioned cases is mainly related with the correlation based calculations from which the phase values are determined. In particular, the robustness of the correlation measurements strongly depends on the number and size of the fringes in the interference patterns recorded at the CCD camera, those fringes characteristics depending on the chosen split-lens distances. .. Among the analysed split-lens configuration, considering the standard deviations shown in Fig. 6, the 0.4 mm separation split-lens is chosen to determine the real phase-voltage look-up table. 


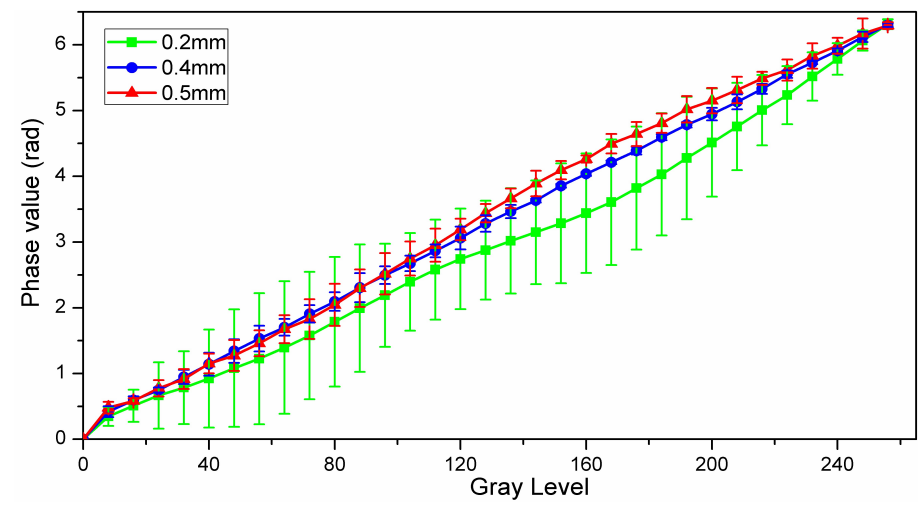

Fig. 6: Average phase modulation (in rad) versus addressed gray level measured using two split lens sectors with center separations of $0.2 \mathrm{~mm}, 0.4 \mathrm{~mm}$ and $0.5 \mathrm{~mm}$.

\section{B. LCoS display spatial shape characterization test}

Once the phase-voltage relation was retrieved, the LCoS surface profile was experimentally characterized by using the method described in section 3. Even though a different property of the studied LCoS is measured, we want to note that the same optical arrangement than that used to measure the phase-voltage look-up table is also used in this section. In fact, the only modification is found in the particular DOE addressed to the LCoS display (the split-lens scheme is replaced by the S-H configuration). As described in section 3, a $4 \times 2$ microlens array was first deployed to the left-up corner of the LCoS screen and the corresponding light-dots patter was recorded at the CCD camera. Afterwards, the displayed DOE was displaced along the $x$ and $y$ directions with steps of 50 pixels. Accordingly, a final $32 \times 16$ light spots pattern (see Fig. 3) was recorded, carrying the spatial information of the full screen. Once the spot array was obtained, the distance of each recorded light spot to its corresponding square center into the reference grid was measured by using centroids calculations. From these data, the LCoS screen derivative function was calculated. Afterwards, the LCoS surface profile was retrieved by applying a numerical integration. However, the surface profile obtained after integration is a discrete function (only contains the characteristics of certain equi-sampled spots of the surface; i.e. 32x16 values). Therefore, a cubic spline interpolation was also applied to retrieve the final continuous LCoS surface profile.

By performing the above-mentioned experimental method, we retrieved a quadratic function for the LCoS surface. This surface profile, presented in radian units, is given in Fig. 7(a). In addition, the same surface phase distribution in $2 \pi$ modulus format is given in Fig. 7(b) for the sake of clarity. We want to note that the experimental achieved quadratic surface can be related with lateral mechanical stresses present at the LCoS screen edges.

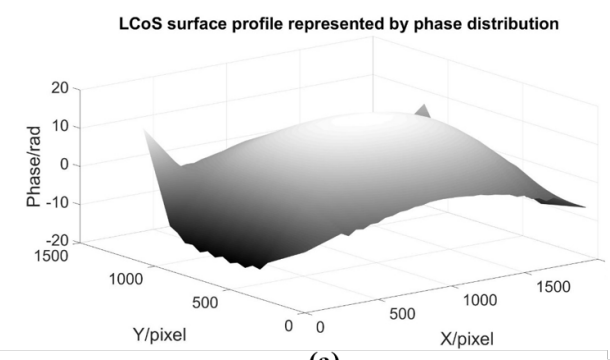

(a)

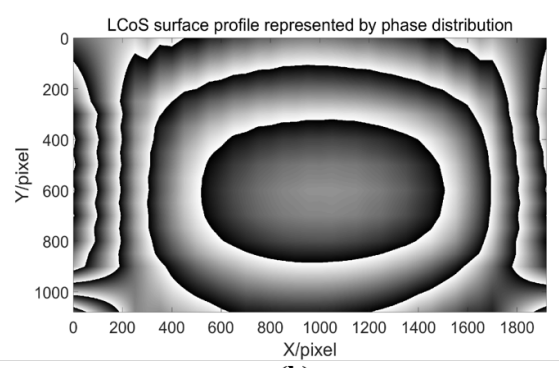

(b)

Fig. 7: (a) LCoS screen surface profile retrieved by using the S-H based method; and (b) The wrapped phase distribution of the LCoS display (modulus of $2 \pi$ ).

To test the validity of the above-retrieved LCoS surface, a further test was performed. In particular, we used the calculated surface in order to correct the undesired LCoS aberration. This was experimentally realized by adding the inverse function of SLM phase distribution (see Fig. 7) and then, applying once again the S-H method to retrieve the new corrected LCoS profile. In the ideal case, by adding the SLM screen inverse phase function, the quadratic phase distribution of the SLM screen should be corrected and the new profile measurement should be significantly flatter. The corrected surface profile was calculated by using the same integration method and the cubic spline interpolation abovediscussed. The obtained corrected LCoS surface profile is provided, in radians, in Fig. 8. Note how Fig. 8(a) presents a 
significantly smoother surface compared to that shown in Fig. 7(a). The phase values in Fig. 8(b) are given in modulus $2 \pi$, and they are almost the same in all pixels except for the surface corners. Hence, we prove how the measured SLM function corrects the LCoS aberration, this providing the validity of the Shack-Hartmann wavefront sensor based method. Finally, we want to emphasize that the flatness loss observed at the four corners in Fig. 8 is caused by the lack of overlapping information in such area during the scanning process (see Video 1). In particular, an extrapolation is used in these sections (four corners) to obtain the surface profile instead of the more accurate cubic spline interpolation implemented to determine the rest surface.

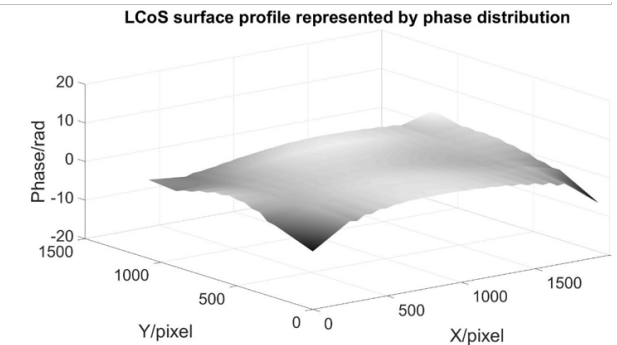

(a)

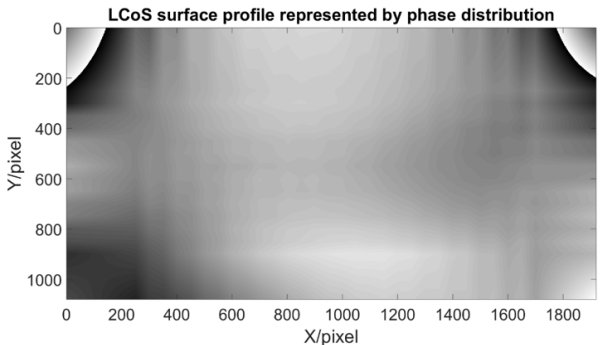

(b)

Fig. 8: (a) Corrected LCoS screen surface profile retrieved by introducing the inverse phase distribution in Fig. 7; and (b)

The wrapped phase distribution of the corrected LCoS surface in modulus of $2 \pi$.

In order to verify the surface modification method more quantitatively, we analysed the phase values along a horizontal line across the whole surface (from the middle pixel of the left edge to the middle pixel of the right edge) in both images shown in Figs. 7(a) and 8(a). The obtained phase values for the horizontal line are shown in Fig. 9 as Curve A (phase values of the original surface; Fig.7) and Curve B (phase values for the corrected surface). Figure 9 clearly shows how the surface quadratic aberration of the LCoS display is significantly corrected. In particular, we obtain in Curve A (original surface) the maximum phase value (15.85 radians) and the minimum phase value (-12.16 radians) at the horizontal line center (around pixel 1000) and at the left-corner (in pixel 0), respectively. The surface variation demonstrated by calculating the peak-to-valley value (PV) is of 28.01 radians. By contrast, the PV of the corrected surface (Curve B) is decreased to 1.28 radians, and the maximum and the minimum phase values are of 3.02 radians and 1.74 radians, respectively. Hence, a $95 \%$ LCoS surface flatness improvement is achieved by the proposed surface modification technique.

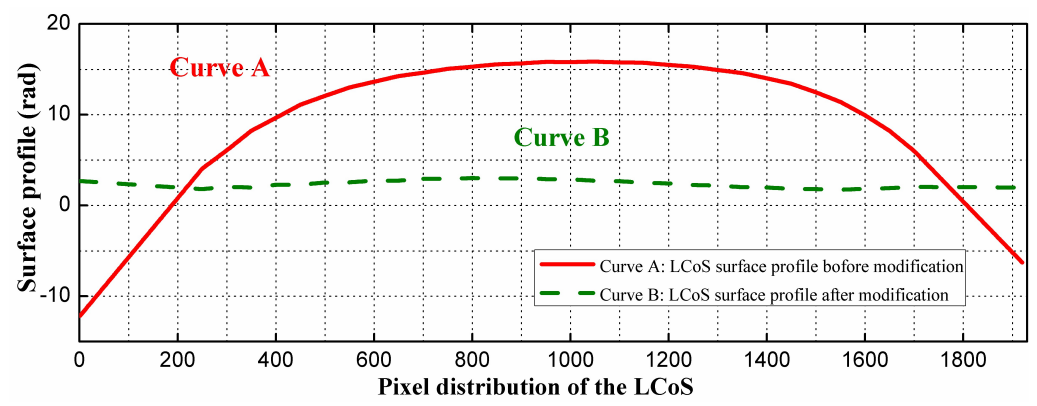

Fig. 9: LCoS surface phase values for a horizontal line in the screen. Data obtained for: (a) The original LCoS surface profile before modification; (b) The LCoS surface profile after correction.

\section{CONCLUSIONS}

In this paper we proposed a Liquid Crystal on Silicon (LCoS) display calibration method based on self-generating lens configurations on the studied device. Our method provides the feasibility to determine the phase-voltage look-up table as well as the surface profile of the LCoS by implementing two different self-generated diffractive lens schemes: a split-lens configuration and a mircolens array (Shack-Hartmann wavefront sensor). In addition, all this can be performed in the same experimental set-up without further modifications. The split-lens configuration allows the generation of a controllable fringes-like interference, from which the phase-voltage relation is retrieved by addressing different constant gray levels to one of the implemented split-lens. Note that this interference based technique is valid even if the liquid 
crystal display presents time-fluctuations of the phase, a non-desired phenomenon present in some LCoS displays. By contrast, the microlens array based method provides the feasibility to determine the LCoS display surface characterization by retrieving the screen spatial phase profile from the light spots distribution generated at the focal plane of the micro-lenses.

We experimentally verified the proposed methods by implementing a particular optical set-up containing the studied LCoS display. In this case, the two proposed lens schemes are experimentally obtained by addressing the corresponding phase distributions at the LCoS display. The phase-voltage look-up table is determined by selecting a $0.4 \mathrm{~mm}$ split-lens separation which provides the best robustness compared to other separation distances analysed. A linear tendency phasevoltage response ranging from $0 \mathrm{rad}$ to $\sim 6.28 \mathrm{rad}$ is demonstrated. Moreover, by implementing the S-H wavefront sensor configuration, we experimentally determined a quadratic LCoS surface profile. Afterward, the surface error was corrected by addressing to the LCoS display the inverse function of the measured quadratic phase measured at the LCoS display. The LCoS screen after applying the correction presents a flat phase distribution. In fact, we demonstrate a screen aberration correction of $95 \%$. The experimental results obtained, and the feasibility of the proposed technique, provides the potential of the method for LCoS characterization processes.

\section{REFERENCES}

[1] Zhang, H., Liu, H., Lizana, A., Xu, W., Campos, J., and Lu, Z., "Methods for the performance enhancement and the error characterization of large diameter ground-based diffractive telescope," Opt. Express, 25(22), 2666226677 (2017).

[2] Mu, Q., Cao, Z., Hu, L., Li, D., and Xuan, L., "Adaptive optics imaging system based on a high resolution liquid crystal on silicon device," Opt. Express, 14(18), 8013-8018 (2006).

[3] Wang, C., Hu, L., Yu, H., Wang, Y., Li, D., Wang, S., Mu, Q., Yang, C., Cao, Z., Lu, X., and Xuan, L., "Wavefront detection method of a single-sensor based adaptive optics system," Opt. Express, 23(16), 2140321413 (2015).

[4] Twientmeyer, K.M., Chipman, R.A., Elsner, A.E., Zhao, Y., and VanNasdale, D., "Mueller matrix retinal imager with optimized polarization conditions," Opt Express, 16(26), 21339-21354 (2008).

[5] Yang, H., Robertson, B., Yu, D., Zhang, Z., and Chu, D.P., "Origin of transient crosstalk and its resolution reduction in phase-only LCoS wavelength selective switches," J Lightwave Technol, 31(22), 3822-3829 (2013).

[6] Robertson, B., Yang, H., Redmond, M.M., Collings, N., Moore, J.R., Liu, J., Jeziorska-Chapman, A.M., Pivnenko, M., Lee, S., Wonfor, A., White, I.H., Crossland, W.A., and Chu, D.P., "Demonstration of multicasting in a $1 \times 9$ LCoS wavelength selective switch," J Lightwave Technol, 32(3), 402-410 (2014).

[7] Suzuki, K., Seno, K., and Ikuma, Y., "Application of waveguide/free-space optics hybrid to ROADM device," J Lightwave Technol, 35(4), 596-606 (2017).

[8] Carles, G., Muyo, G., Bosch, S., and Harvey, A.R., "Use of a spatial light modulator as an adaptable phase mask for wavefront coding," J Mod Opt, 57(10), 893-900(2010).

[9] Márquez, J.L., Fernández, E.J., Prieto, P.M., and Artal, P., "Chromatic aberration control with liquid crystal spatial phase modulators," Opt Express, 25(9), 9793-9801(2017).

[10]Zheng, X., Lizana, A., Peinado, A., Ramírez, C., Martínez, J.L., Márquez, A., Moreno, I., and Campos, J., "Compact LCoS-SLM based polarization pattern beam generator," J Lightwave Technol, 33(10), 2047-2055 (2015).

[11]Lizana, A., Vargas, A., Turpin, A., Ramírez, C., Estevez, I., and Campos, J., "Shaping light with split lens configurations," J Opt, 18(10), 105605 (2016).

[12] Rodrigo, J., Alieva, T., Abramochkin, E., and Castro, I., "Shaping of light beams along curves in three dimensions," Opt Express, 21(18), 20544-20555 (2013).

[13] Rodrigo, J., and Alieva, T., "Freestyle 3D laser traps: tools for studying light-driven particle dynamics and beyond," Optica, 2(9), 812-815 (2015).

[14] Martín-Badosa, E., Montes-Usategui, M., Carnicer, A., Andilla, J., Pleguezuelos, E., and Juvells, I., "Design strategies for optimizing holographic optical tweezers set-ups," J Opt A-Pure Appl Opt, 9(8), S267 (2007).

[15] Bowman, R.W., Wright, A.J., and Padgett, M.J., "An SLM-based Shack-Hartmann wavefront sensor for aberration correction in optical tweezers," J Opt, 12(12), 124004 (2010).

[16] Tu, H., Cheng, C., and Chen, M., "Optical image encryption based on polarization encoding by liquid crystal spatial light modulators," J Opt A-Pure Appl Opt, 6(6), 524 (2004). 
[17]Campos, J., López-Coronado, O., Iemmi, C., Escalera, J.C., Gimeno, R., and Yzuel, M.J., "Multiplexed lenses written onto a liquid crystal display to increase depth of focus," Proc. SPIE 6311, 63110Q (2006).

[18] Márquez, A., Iemmi, C., Campos, J., Escalera, J.C., and Izuel, M.J., "Programmable apodizer to compensate chromatic aberration effects using a liquid crystal light modulator," Opt Express, 13(3), 716-730 (2005).

[19]Zhang, Z., You, Z., and Chu, D., "Fundamentals of phase-only liquid crystal on silicon (LCoS) devices," Light Sci Appl, 3(10), e213 (2014).

[20] Yang, L., Xia, J., Chang, C.L., Yang, Z.M., and Chen, J.H., "Nonlinear dynamic phase response calibration by digital holographic microscopy," Appl Opt, 54(25), 7799-7806 (2015).

[21]Lizana, A., Moreno, I., Márquez, A., Iemmi, C., Fernandez, E., Campos, J., and Yzuel, M.J., "Time fluctuations of the phase modulation in a liquid crystal on silicon display: characterization and effects in diffractive optics," Opt Express, 26(21), 16711-16722 (2008).

[22] Ramirez, C., Karakus, B., Lizana, A., and Campos, J., "Polarimetric method for liquid crystal displays characterization in presence of phase fluctuations," Opt Express, 21(3), 3182-3192 (2013).

[23] Soutar, C., Monroe, S.E., and Knopp, J., "Measurement of the transmittance of the Epson liquid crystal television," Opt Eng, 33(4), 1061-1069 (1994).

[24]Bergeron, A., Gauvin, J., Gagnon, F., Gingras, D., Arsenault, H.H., and Doucet, M., "Phase calibration and applications of a liquid crystal spatial light modulator," Appl Opt, 34(23), 5133-5139 (1995).

[25] Engström, D., Milewski, G., Bengtsson, J., and Galt, S., "Diffraction-based determination of the phase modulation for general spatial light modulators," Appl Opt, 45(28), 7195-7204 (2006).

[26]Zhang, Z., Lu, G., and Francis, T.S., "Simple method for measuring phase modulation in liquid crystal televisions," Opt Eng, 33(9), 3018-3022 (1994).

[27] Martínez, F.J., Márquez, A., Gallego, S., Francés, J., Pascual, I., and Beléndez, A., "Retardance and flicker modeling and characterization of electro-optic linear retarders by averaged Stokes polarimetry," Opt Lett, 39(4), 1011-1014 (2014).

[28] Martínez, F.J., Márquez, A., Gallego, S., Ortuño, M., Francés, J., Beléndez, A., and Pascual, I., "Averaged Stokes polarimetry applied to evaluate retardance and flicker in PA-LCoS devices," Opt Express, 22(12), 15064-15074 (2014).

[29] Martínez, J.L., Fernández, E.J., Prieto, P.M., and Artal, P., "Interferometric method for phase calibration in liquid crystal spatial light modulator using a self-generated diffraction-grating," Opt Express, 24(13), 1415914171 (2017).

[30] Márquez, A., Moreno, I., Iemmi, C., Lizana, A., Campos, J., and Yzuel, M.J., "Mueller-Stokes characterization and optimization of a liquid crystal on silicon display showing depolarization," Opt Express, 16(3), 1669-1685 (2008).

[31]Lizana, A., Moreno, I., Iemmi, C., Márquez, A., Campos, J., and Yzuel, M.J., "Time-resolved Mueller matrix analysis of a liquid crystal on silicon display," Appl Opt, 47(23), 4267-4274 (2008).

[32] Lizana, A., Márquez, A., Lobato, L., Rodagne, Y., Moreno, I., Iemmi, C., and Campos, J., "The minimum Euclidean distance principle applied to improve the modulation diffraction efficiency in digitally controlled spatial light modulators," Opt Express, 18(10), 10581-10593 (2010).

[33] García-Márquez, J., López, V., González-Vega, A., and Noé, E., "Flicker minimization in an LCoS spatial light modulator," Opt Express, 20(8), 8431-8441 (2012).

[34]Lopez-Quesada, C., Andilla, J., and Martin-Badosa, E., "Correction of aberration in holographic optical tweezers using a Shack-Hartmann sensor," Appl Opt, 48(6), 1084-1090 (2009).

[35] Vargas, J., Uribe-Patarroyo, N., Quiroga, J.A., Alvarez-Herrero, A., and Belenguer, T., "Optical inspection of liquid crystal variable retarder inhomogeneities," Appl Opt, 49(4), 568-574 (2010).

[36] Yun, Z., Liu, J., He, Z., and Li, Y., "Measurement of the thickness uniformity of liquid crystal layer," Appl Opt, 36(34), 9109-9110 (1997).

[37]Lobato, L., Márquez, A., Lizana, A., Moreno, I., and Campos, J., Characterization of a Parallel Aligned Liquid Crystal on Silicon and its application on a Shack-Hartmann sensor," Proc. SPIE 7797, 77970Q-1 (2010).

[38] Schiegerling, J., and Dehoog, E., Problems testing diffractive intraocular lenses with Shack-Hartmann sensors," Appl Opt, 47(25), 4548-4554 (2008).

[39] Smith, D.G., and Greivenkamp, J.E., Generalized method for sorting Shack-Hartmann spot patterns using local similarity," Appl Opt, 49(16), D62-D68 (2010). 